\title{
Prevalence and correlates of different smoking bans in homes and cars among smokers in six countries of the EUREST-PLUS ITC Europe Surveys
}

\author{
Marcela Fu ${ }^{1,2,3}$, Yolanda Castellano ${ }^{1,2}$, Olena Tigova ${ }^{1,2}$, Christina N. Kyriakos ${ }^{4,5}$, Geoffrey T. Fong ${ }^{6,7}$, Ute Mons ${ }^{8}$, Witold A.

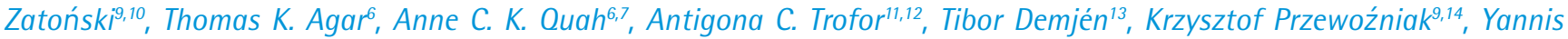 \\ Tountas $^{15}$, Constantine I. Vardavas ${ }^{4,5}$, Esteve Fernández ${ }^{1,2,3}$, on behalf of the EUREST-PLUS consortium*
}

\section{ABSTRACT}

INTRODUCTION Second-hand smoke exposure has decreased in a number of countries due to widespread smoke-free legislation in public places, but exposure is still present in private settings like homes and cars. Our objective was to describe to what extent smokers implement smoking rules in these settings in six European Union (EU) Member States (MS).

METHODS A cross-sectional survey was conducted with a nationally representative sample of adult smokers from Germany, Greece, Hungary, Poland, Romania and Spain (ITC six European countries survey, part of the EUREST-PLUS Project). We analysed data from 6011 smokers regarding smoking rules in their homes and in cars with children (no rules, partial ban, total ban). We described the prevalence of smoking rules by EU MS and several sociodemographic and smoking characteristics using prevalence ratios (PR) and 95\% confidence intervals (CI) derived from Poisson regression models.

RESULTS In homes, $26.5 \%$ had a total smoking ban (from 13.1\% in Spain to $35.5 \%$ in Hungary), $44.7 \%$ had a partial ban (from $41.3 \%$ in Spain to $49.9 \%$ in Greece), and $28.8 \%$ had no-smoking rules (from $20.2 \%$ in Romania to $45.6 \%$ in Spain). Prevalence of no-smoking rules in cars with children was $16.2 \%$ (from $11.2 \%$ in Germany to $20.4 \%$ in Spain). The correlates of not restricting smoking in homes and cars included: low education ( $\mathrm{PR}=1.51 ; 95 \% \mathrm{CI}$ : $1.20-1.90$ and $\mathrm{PR}=1.55$; 95\%CI: $1.09-2.20)$, smoking $>30$ cigarettes daily ( $\mathrm{PR}=1.53$; 95\%CI: 1.10-2.14 and $\mathrm{PR}=2.66$; 95\%CI: $1.40-5.05)$ and no attempts to quit ever ( $\mathrm{PR}=1.18$; 95\%CI: $1.06-1.31$ and $\mathrm{PR}=1.28$; 95\%CI: $1.06-1.54)$. CONCLUSIONS Among smokers in six EU MS, no-smoking rules were more prevalent in homes than in cars with children. Whilst awareness about the health effects of exposure to tobacco smoke on children seemed to be high, more research is needed to better understand the factors that promote private smoke-free environments.

\author{
AFFILIATION \\ 1 Catalan Institute of Oncology, Catalonia, Spain \\ 2 Bellvitge Biomedical Research Institute \\ (IDIBELL), Catalonia, Spain \\ 3 University of Barcelona, Catalonia, Spain \\ 4 European Network on Smoking and Tobacco \\ Prevention (ENSP), Brussels, Belgium \\ 5 University of Crete (UoC), Heraklion, Greece \\ 6 University of Waterloo (UW), Waterloo, Canada \\ 7 Ontario Institute for Cancer Research, \\ Toronto, Canada \\ 8 Cancer Prevention Unit and WHO \\ Collaborating Centre for Tobacco Control, \\ German Cancer Research Centre (DKFZ), \\ Heidelberg, Germany \\ 9 Health Promotion Foundation (HPF), \\ Warsaw, Poland \\ 10 European Observatory of Health \\ Inequalities, The President Stanisław \\ Wojciechowski State University of Applied \\ Sciences, Kalisz, Poland \\ 11 University of Medicine and Pharmacy \\ 'Grigore T. Popa' Iasi, Iasi, Romania \\ 12 Aer Pur Romania, Bucharest, Romania \\ 13 Smoking or Health Hungarian Foundation \\ (SHHF), Budapest, Hungary \\ 14 Maria Skłodowska-Curie Institute- \\ Oncology Center, Warsaw, Poland \\ 15 National and Kapodistrian University of \\ Athens (UoA), Athens, Greece
}

\section{CORRESPONDENCE TO}

Marcela Fu. Tobacco Control Unit, Catalan Institute of Oncology, Av. Granvia de L'Hospitalet, 199-203, 08908 L'Hospitalet de Llobregat, Catalonia, Spain. E-mail:mfu@iconcologia.net

KEYWORDS

voluntary smoke-free regulation, private settings, smokers, Europe, WHO FCTC

Received: 8 June 2018

Revised: 18 August 2018

Accepted: 3 September 2018

\section{INTRODUCTION}

Second-hand tobacco smoke is one of the most widespread air pollutants in indoor environments ${ }^{1}$.
Exposure to it has been linked to several health outcomes, including respiratory asthma, infections of the lower respiratory tract, otitis media and sudden 
infant death syndrome, as well as ischaemic heart disease and lung cancer in adults ${ }^{1}$. The World Health Organization (WHO) Framework Convention on Tobacco Control (FCTC), an international health treaty, promotes smoke-free environments to achieve effective protection from the hazards of secondhand tobacco smoke. Article 8 of the FCTC calls upon Parties to implement measures for protection from exposure to tobacco smoke in public places, including but not limited to indoor workplaces, public transportation, indoor public places, and other public places $^{2}$. Guidelines for the implementation of Article 8 have been developed further to assist countries in the adoption and implementation of smoke-free measures, as well as in identifying the key elements of their smoke-free legislation ${ }^{3}$.

Ratification of the WHO FCTC is associated with the accelerated implementation of key demand-reduction measures, including smoke-free laws ${ }^{4,5}$, with evidence of significant dose-respondent decreases in smoking prevalence and highest-level implementation of these measures $^{6}$. Since 2004, many countries have adopted complete national smoking bans in public places, which has resulted in benefits to both non-smokers (less second-hand smoke exposure) and smokers (tend to smoke less, have greater cessation success, and experience more confidence in their ability to quit $)^{7}$. However, only $16 \%$ of the world's population is covered by comprehensive smoke-free laws ${ }^{7}$. Furthermore, these policies at the national level have only been adopted within the public domain and do not usually apply to private settings, such as homes and cars, where exposure to second-hand tobacco smoke is still common ${ }^{8,9}$. Nevertheless, some studies indicate that smoke-free legislation has had unexpected effects in promoting private smoke-free settings ${ }^{10,11}$. It has been hypothesised that public policies affect a variety of psychosocial and behavioural variables ${ }^{12}$. We hypothesise that the implementation of robust smoke-free legislation in public places will lead to the subsequent cessation of smoking in public places; this will in a second stage lead to changes in psychological mediators (e.g. awareness of the health effects of second-hand tobacco smoke) and in a third stage to behavioural changes, such as the establishment of voluntary tobacco-free policies in private settings.

Little is known on the extent to which smokers implement smoke-free bans within their own private settings. Thus, the objective of this study was to assess existing smoking rules and their correlates in smokers' private settings, such as homes and cars, in six European countries.

\section{METHODS}

\section{Design}

This study is part of the European Commission Horizon-2020 funded study entitled European Regulatory Science on Tobacco: Policy implementation to reduce lung diseases (EUREST-PLUSHCO-06-2015; https://eurestplus.eu/), with main objective to monitor and assess the impact of the ratification of the WHO FCTC at the European level, through the implementation of the Tobacco Products Directive $(2014 / 40 / \mathrm{EU})^{13}$. To achieve this goal, a prospective cohort study of approximately 6000 smokers began using representative samples of smokers in each of the following six European Union (EU) Member States (MS): Germany $(n=1003)$, Greece $(n=1000)$, Hungary $(n=1000)$, Poland $(n=1006)$, Romania $(n=1001)$, and Spain $(n=1001)$.

This cohort survey, the ITG six European countries survey (ITC 6E Survey), is part of the ongoing International Tobacco Control Project Policy Evaluation (ITG) Project (www.itcproject. org/), which aims at tracking and comparing the impact of national-level tobacco policies among representative samples of adult smokers in 29 countries. The methods used in the ITC $6 \mathrm{E}$ Survey are explained elsewhere ${ }^{14}$. Briefly, samples of adult ( $\geqslant 18$ years old) current smokers (having smoked $>100$ cigarettes in their lifetime and having smoked at least once in the past 30 days) were recruited using probability sampling methods, representative of all geographical regions in each EU MS. Eligible households were randomly selected using a randomwalk method, and a household was considered eligible if it included at least one eligible smoker. Where available, both one male and one female smoker were selected from each household using the last birthday method ${ }^{15}$. Baseline data (i.e. Wave 1 of the ITC 6E Survey) were collected over a month, in each EU MS, between June and September 2016. After informed consent was provided, a computer-assisted personal interview was conducted. Participants received remuneration as an incentive for their participation. The study protocol was approved by 
an ethics committee in each participating country and partnering institutions.

This report is a cross-sectional baseline analysis of data collected from Wave 1 on the degree to which smoking rules are implemented in private indoor settings, namely, in smokers' homes and in their cars in the presence of children.

\section{Measures}

\section{Smoking rules in homes}

Rules in homes were ascertained by the question: "Which of the following statements best describes smoking inside your home? I mean inside your house or dwelling and not on the balcony, terrace, or other outdoor areas'. The possible answers were: 'smoking allowed anywhere inside the home', 'smoking allowed in some rooms inside the home', 'smoking never allowed anywhere inside the home', and 'smoking not allowed inside home except under special circumstances'. These possible answers were re-coded as 'no rules' (first possible answer), 'partial ban' (second and last possible answers), and 'total ban' (third possible answer).

\section{Smoking rules in cars}

Rules in cars were ascertained with the question: 'What are the rules about smoking in your car or cars when there are children in the car?'. The possible answers were: 'smoking never allowed in any car', 'smoking allowed sometimes or in some cars', and 'smoking allowed in all cars'. These possible answers were re-coded as 'total ban', 'partial ban' and 'no rules', respectively. Answers 'do not have a car' or 'never have children in car' were excluded from the analyses.

\section{Analysis}

We describe the prevalence of different smoking rules (no rules, partial and total smoking ban) in homes and in cars of smokers by EU MS, by several sociodemographic (sex, age, educational level - a country-specific variable standardised and categorised as low, intermediate, and high -, partner's smoking status, having children) and by smoking characteristics (cigarettes smoked per day - CPD, nicotine dependence measured by the Heaviness of Smoking Index ${ }^{16}$, and attempts to quit smoking). We also conducted pairwise comparisons of smoking rules (partial vs total ban; no rules vs total ban) according to all independent variables using prevalence ratios (PR) and their 95\% confidence intervals (CI) derived from Poisson regression models with robust variance, adjusting for all independent variables. All the analyses incorporated the weights derived from the complex sampling design. We used Stata v.13 for all analyses.

\section{RESULTS}

\section{Smoking rules at home}

Among all smokers across the six countries, 26.5\% reported a total smoking ban in their home, with Hungary having the highest prevalence (35.5\%) and Spain the lowest (13.1\%); see Figure 1 and Supplementary Table 1. On the other hand, 28.8\% of smokers across the six countries had no-smoking rules in their homes, varying from $20.2 \%$ in Romania to $45.6 \%$ in Spain. Overall, the prevalence of a total smoking ban in homes was similar in both sexes, but was significantly lower among participants aged 55 years or older and among those with lower educational level (Table 1). The prevalence of a total

\section{Figure 1. Prevalence of smoking rules in smokers' homes and cars with children, 2016}
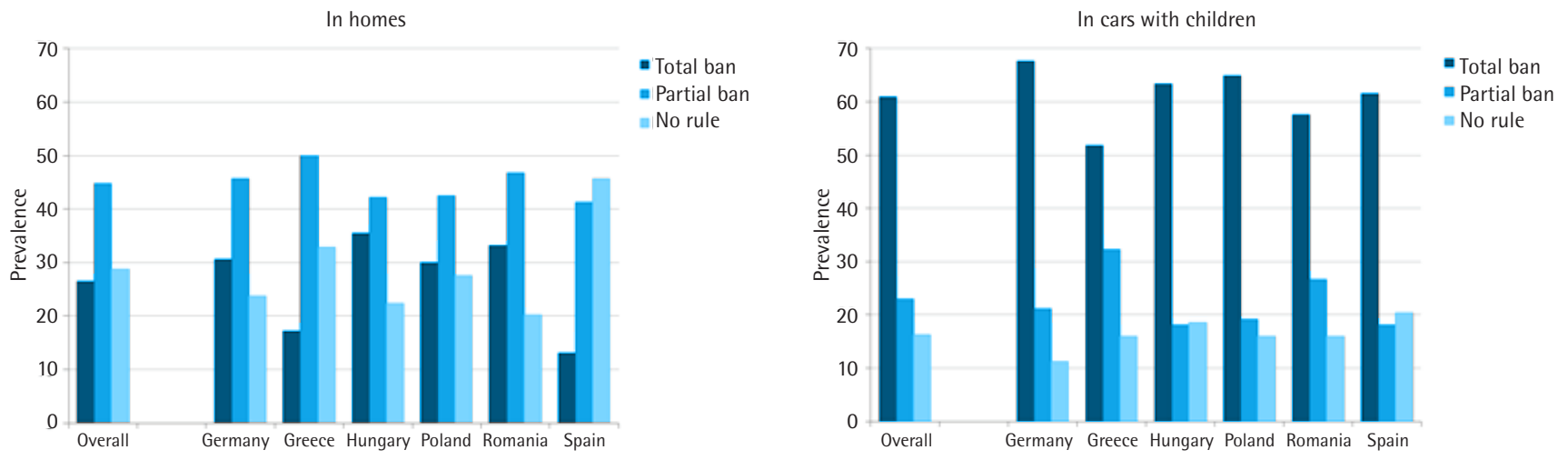
Table 1. Prevalence of smoking rules in smokers' homes, according to sociodemographic and smoking characteristics, 2016

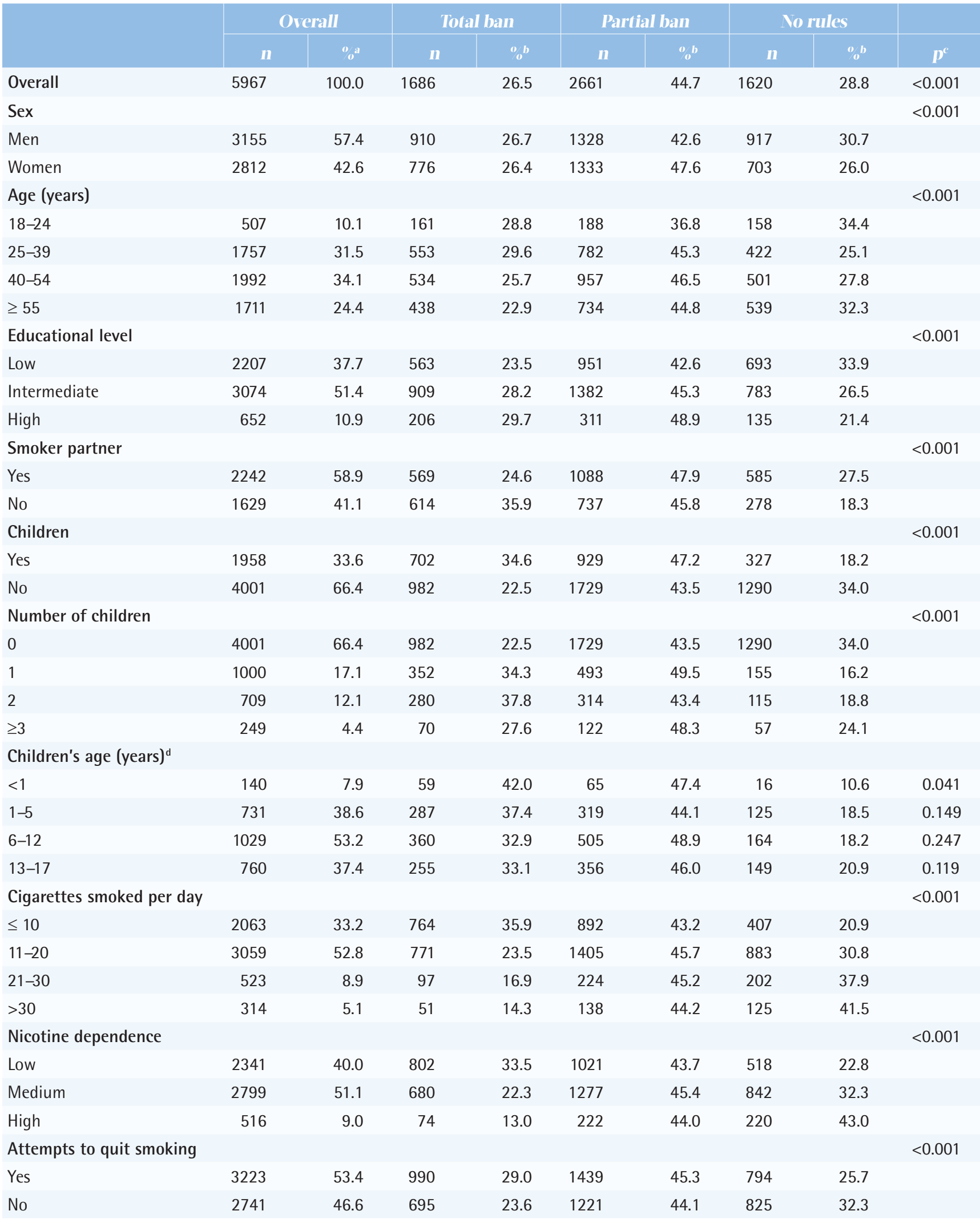

a Weighted percentages per column. b Weighted percentages per row. $c X^{2}$ test. $d$ Multiple response. 
smoking ban in homes was higher among smokers with a non-smoker partner and among those with more children. Forty-two per cent of smokers who had children less than one year of age had a total smoking ban in their homes, varying from $11.4 \%$ in Spain to $56.6 \%$ in Romania (Supplementary Table 2). Additionally, there was a higher prevalence of total smoking bans among homes of smokers who reported to smoke less, were least nicotine dependent, and who had ever attempted to quit smoking (Table 1).
Information for each EU MS is provided in the Supplementary Table 2.

We further assessed the association of smoking rules in homes (partial vs total ban; no rules vs total ban) with different independent variables, where prevalence ratios $>1$ indicate less smoking rules in homes (Table 2). Compared to smokers from Germany, the countries where smokers were more likely to have a partial smoking ban or no-smoking rules in their homes were Spain $(\mathrm{PR}=1.40,95 \%$

Table 2. Comparison of smoking rules (partial ban vs total ban; no rules vs total ban) in smokers' homes, according to independent variables, 2016

\begin{tabular}{|c|c|c|c|c|c|c|}
\hline & \multicolumn{3}{|c|}{ Partial ban us Total ban } & \multicolumn{3}{|c|}{ Vo rules us Total ban } \\
\hline & PR & $95 \% \mathrm{CI}$ & $p^{\mathrm{a}}$ & $P R$ & $95^{\circ} \% \mathrm{CI}$ & $p^{2}$ \\
\hline \multicolumn{7}{|l|}{ Country } \\
\hline Germany & 1 & - & & 1 & - & \\
\hline Greece & 1.23 & $1.07-1.42$ & 0.005 & 1.68 & $1.29-2.19$ & $<0.001$ \\
\hline Hungary & 0.94 & $0.80-1.10$ & 0.417 & 1.12 & $0.85-1.47$ & 0.422 \\
\hline Poland & 0.99 & $0.85-1.15$ & 0.883 & 1.33 & $1.04-1.71$ & 0.026 \\
\hline Romania & 1.07 & $0.92-1.24$ & 0.394 & 1.27 & $0.97-1.65$ & 0.082 \\
\hline Spain & 1.40 & $1.23-1.60$ & $<0.001$ & 2.38 & $1.89-2.98$ & $<0.001$ \\
\hline \multicolumn{7}{|l|}{ Sex } \\
\hline Men & 1 & - & & 1 & - & \\
\hline Women & 1.09 & $1.04-1.15$ & 0.001 & 1.12 & $1.02-1.22$ & 0.014 \\
\hline \multicolumn{7}{|l|}{ Age (years) } \\
\hline $18-24$ & 1.04 & $0.87-1.24$ & 0.692 & 1.15 & $0.90-1.47$ & 0.259 \\
\hline $25-39$ & 1.04 & $0.94-1.15$ & 0.445 & 1.01 & $0.88-1.15$ & 0.898 \\
\hline $40-54$ & 1.04 & $0.95-1.14$ & 0.356 & 0.96 & 0.85-1.09 & 0.558 \\
\hline$\geq 55$ & 1 & - & & 1 & - & \\
\hline \multicolumn{7}{|c|}{ Educational level } \\
\hline Low & 1.05 & $0.94-1.18$ & 0.370 & 1.51 & $1.20-1.90$ & 0.001 \\
\hline Intermediate & 1.00 & $0.90-1.12$ & 0.995 & 1.21 & $0.97-1.51$ & 0.097 \\
\hline High & 1 & - & & 1 & - & \\
\hline \multicolumn{7}{|c|}{ Smoker partner } \\
\hline Yes & 1.18 & $1.10-1.27$ & $<0.001$ & 1.49 & $1.33-1.68$ & $<0.001$ \\
\hline No & 1 & - & & 1 & - & \\
\hline \multicolumn{7}{|l|}{ Children } \\
\hline Yes & 1 & - & & 1 & - & \\
\hline No & 1.19 & $1.10-1.29$ & $<0.001$ & 1.64 & $1.44-1.86$ & $<0.001$ \\
\hline \multicolumn{7}{|c|}{ Cigarettes smoked per day } \\
\hline$\leq 10$ & 1 & - & & 1 & - & \\
\hline $11-20$ & 1.22 & $1.12-1.34$ & $<0.001$ & 1.41 & $1.20-1.64$ & $<0.001$ \\
\hline $21-30$ & 1.22 & $1.06-1.42$ & 0.007 & 1.52 & $1.22-1.90$ & $<0.001$ \\
\hline$>30$ & 1.18 & $0.94-1.48$ & 0.147 & 1.53 & $1.10-2.14$ & 0.013 \\
\hline
\end{tabular}


Table 2. continued

\begin{tabular}{|c|c|c|c|c|c|c|}
\hline & \multicolumn{3}{|c|}{ Partial ban vs Total ban } & \multicolumn{3}{|c|}{ No rules vs Total ban } \\
\hline & PR & $95^{\circ} \circ \mathrm{CI}$ & $p^{a}$ & PR & $95 \% \mathrm{CI}$ & $p^{a}$ \\
\hline \multicolumn{7}{|c|}{ Nicotine dependence } \\
\hline Low & 1 & - & & 1 & - & \\
\hline Medium & 1.03 & 0.94-1.13 & 0.486 & 1.17 & $1.01-1.36$ & 0.038 \\
\hline High & 1.15 & $0.96-1.39$ & 0.128 & 1.33 & $1.01-1.76$ & 0.044 \\
\hline \multicolumn{7}{|c|}{ Attempts to quit smoking } \\
\hline Yes & 1 & - & & 1 & - & \\
\hline No & 1.06 & 0.99-1.13 & 0.090 & 1.18 & $1.06-1.31$ & 0.002 \\
\hline
\end{tabular}

Prevalence ratios (PR) are derived from Poisson regression models, adjusted for all independent variables. a $x^{2}$ test.

CI: $1.23-1.60$ for partial vs total smoking ban; $\mathrm{PR}=2.38$, 95\% CI: 1.89-2.98 for no-smoking rules vs total smoking ban) and Greece (PR=1.23, 95\% CI: 1.07-1.42 for partial vs total smoking ban; $\mathrm{PR}=1.68$, 95\% CI: 1.29-2.19 for no-smoking rules vs total smoking ban). The variables significantly associated with not having smoking rules in homes were: female, low educational level, partner who smokes, no children, more CPD, more nicotine dependent, and never attempted to quit smoking (Table 2).

\section{Smoking rules in cars with children}

Overall, $60.9 \%$ of smokers stated that they had a total smoking ban in their cars when children were present, $22.9 \%$ stated having a partial ban, and $16.2 \%$ stated having no-smoking rules at all. Smoking rules varied across EU MS, with Greece having the lowest prevalence for cars with a total smoking ban (51.8\%) and Germany having the highest prevalence (67.7\%); see Figure 1 and Supplementary Table 1. Analyses examining associations with other independent variables showed that cars with total smoking bans were more prevalent among female smokers, and this increased with age and with educational level.
The prevalence of total smoking bans in cars was also higher among smokers with a non-smoker partner, with more children, among those who smoked fewer CPD, with lower nicotine dependence, and with any ever attempt to quit smoking (Table 3). Overall, smokers consuming $>30$ CPD were the only group with a prevalence of a total smoking ban in cars of less than $50 \%$ (Table 3 ), varying from $32.6 \%$ in Greece to $59.0 \%$ in Spain. Information by country is provided in the Supplementary Table 3.

Further analysis comparing smoking rules in cars (partial vs total ban, and no rules vs total ban) adjusting for potential confounders showed that, compared to Germany, only smokers from Greece were more likely to have a partial smoking ban rather than a total ban (PR=1.67; 95\% CI: 1.20-2.34; Table 4). Smokers from all countries, except Hungary, had a higher prevalence of not having smoking rules than having a total smoking ban in cars, compared to Germany. As shown in Table 4, having no-smoking rules in cars was more likely among smokers at younger ages, low educational level, who smoked more CPD, with medium nicotine dependence, and with no attempts to quit smoking ever.

Table 3 Prevalence of smoking rules in smokers' cars with children, according to sociodemographic and smoking characteristics, 2016

\begin{tabular}{|c|c|c|c|c|c|c|c|c|c|}
\hline & & & & & & an & & & \\
\hline & n & $\%$ & n & $\%^{b}$ & n & $\%^{b}$ & n & $\% b$ & $p^{c}$ \\
\hline Overall & 3885 & 100 & 2409 & 60.9 & 839 & 22.9 & 637 & 16.2 & $<0.001$ \\
\hline Sex & & & & & & & & & $<0.001$ \\
\hline Men & 2060 & 57.4 & 1211 & 57.9 & 481 & 24.3 & 368 & 17.8 & \\
\hline Women & 1825 & 42.6 & 1198 & 64.9 & 358 & 20.9 & 269 & 14.2 & \\
\hline
\end{tabular}


Table 3. continued

\section{Overall}

Age (years)

$18-24$

25-39

$40-54$

$\geq 55$

Educational level

Low

Intermediate

High

Smoker partner

Yes

No

Children

Yes

No

Number of children

0

1

2

$\geq 3$

Children's age (years) ${ }^{\mathrm{d}}$

$<1$

$1-5$

6-12

13-17

Cigarettes smoked per day

$\leq 10$

$11-20$

$21-30$

$>30$

Nicotine dependence

Low

Medium

High

Attempts to quit smoking

Yes

No

1268

2091

504

1611

1196

1467

2412

522

559

1417

1953

189

312

2150

1734

$\begin{array}{rrr}272 & 10.1 & 151 \\ 1198 & 31.5 & 729 \\ 1408 & 34.1 & 856 \\ 1007 & 24.4 & 673\end{array}$

\section{Total ban}

673

$37.7 \quad 763$

$51.4 \quad 1300$

$10.9 \quad 332$

$58.9 \quad 993$

$41.1 \quad 780$

$33.6 \quad 925$

$66.4 \quad 1482$

62.5

51.7

61.7

59.1

66.3

175

$58.8 \quad 269$

$61.2 \quad 447$

$65.1 \quad 116$

65

$\begin{array}{rr}25.8 & 56 \\ 21.9 & 210 \\ 25.5 & 212 \\ 18.5 & 159\end{array}$

$22.1 \quad 236$

$22.9 \quad 344$

$24.2 \quad 56$

0.470

$60.9 \quad 351$

$63.6 \quad 238$

$22.8 \quad 267$

$21.5 \quad 178$

0.354

$62.5 \quad 317$

22.4

225

15.1

23.0

411

17.0

0.047

$\begin{array}{rrrrr}2412 & 66.4 & 1482 & 60.0 & 519 \\ 761 & 17.1 & 477 & 61.7 & 154 \\ 545 & 12.1 & 361 & 66.6 & 118 \\ 161 & 4.4 & 87 & 53.5 & 45\end{array}$

$\begin{array}{lll}96 & 7.9\end{array}$

$38.6 \quad 335$

$53.2 \quad 483$

$37.4 \quad 357$

$59.4 \quad 18$

$63.7 \quad 110$

$61.4 \quad 191$

$64.0 \quad 115$

$33.2 \quad 1040$

$72.2 \quad 224$

$52.8 \quad 1130$

$56.2 \quad 477$

$8.9 \quad 167$

$55.4 \quad 76$

39.5

61

$5.1 \quad 70$

$1619 \quad 40.0 \quad 1127$

$68.2 \quad 297$

$54.9 \quad 419$

$45.7 \quad 93$

$51.1 \quad 967$

132

$9.0 \quad 132$

$23.0 \quad 411$

17.0

$21.2 \quad 130$

17.1

$21.7 \quad 66$

11.7

$29.6 \quad 29$

17.0

a Weighted percentages per column. $b$ Weighted percentages per row. $c X^{2}$ test. $d$ Multiple response. 
Table 4. Comparison of smoking rules (partial ban vs total ban; no rules vs total ban) in smokers' cars in presence of children according to independent variables, 2016

\begin{tabular}{|c|c|c|c|c|c|c|}
\hline & \multicolumn{3}{|c|}{ Partial ban vs Total ban } & \multicolumn{3}{|c|}{ Vo rules vs Total ban } \\
\hline & $P R$ & $95^{\circ} \circ \mathrm{CI}$ & $p^{a}$ & PR & $95^{\circ} \% \mathrm{CI}$ & $p^{3}$ \\
\hline
\end{tabular}

\section{Country}

Germany

Greece

Hungary

1.67

1.20-2.34

0.003

1

Poland

0.98

0.67-1.43

0.921

1.68

1.10-2.59

0.018

Romania

0.98

0.67-1.44

0.912

0.94-2.35

0.093

Spain

1.44

0.98-2.11

0.061

1.12-2.88

0.015

Sex

0.65-1.54

0.993

1.18-2.94

0.007

1.00

1.86

1.34-3.16

0.001

Men

Women

1

Age (years)

18-24

1.65

1.29

$1.01-1.65$

0.025

0.110

0.87

0.73-1.04

0.125

25-39

1.45

1.19-1.76

0.039

1.04-1.77

0.033

40-54

$<0.001$

1.64

0.024

$\geq 55$

1

Educational level

Low

1.20

0.92-1.57

0.176

1.55

1.09-2.20

0.014

Intermediate

0.88-1.41

0.381

0.87-1.72

0.249

High

Smoker partner

Yes

$0.89-1.23$

0.606

1.10

0.88-1.37

0.411

No

1

1

Children

Yes

1

No

1.13

$0.94-1.34$

0.194

1.22

$0.99-1.50$

0.061

Cigarettes smoked per day

$\leq 10$

$11-20$

1.74

$1.33-2.27$

$<0.001$

1.46

$1.06-1.99$

0.019

21-30

1.30

0.88-1.90

0.184

$1.19-2.75$

0.005

$>30$

1.45

$0.85-2.46$

0.169

1.81

$1.40-5.05$

0.003

Nicotine dependence

Low

Medium

1

High

1.01

0.79-1.29

0.917

1.40

1.06-1.85

0.018

1.47

$0.92-2.33$

0.105

1.25

$0.72-2.16$

0.423

Attempts to quit smoking

Yes

1

1.12

$0.96-1.31$

0.141

1.28

$1.06-1.54$

0.009

Prevalence ratios (PR) are derived from Poisson regression models, adjusted for all independent variables. a $\chi^{2}$ test. 


\section{DISCUSSION}

More than a quarter of smokers surveyed across six EU MS reported having a total smoking ban in their homes; a similar proportion of smokers had no smoking rules. Prevalence of total smoking ban in homes varied across countries, from $13.1 \%$ in Spain to $35.5 \%$ in Hungary. These findings are consistent with data from other studies conducted in European countries within the ITC Project ${ }^{17-20}$ that indicated cross-country differences in the prevalence of smoke-free homes, varying from $13 \%$ in Scotland in 2007 to $38.2 \%$ in Germany in 2009 . The later studies also indicated an increased prevalence of smokefree homes in European countries over two survey waves $^{17-20}$.

A different situation was found in cars when children were present where $61 \%$ of smokers had a total smoking ban and only $16 \%$ had no rules, with less heterogeneity observed across countries, varying from $51.8 \%$ in Greece to $67.7 \%$ in Germany. Data from other ITC studies conducted between 2007 and 2008 in France, Germany and the Netherlands indicated generally lower support for smoking bans in cars when children are present $(41 \%, 48 \%, 64 \%$, respectively $)^{21}$, probably due to the time that has elapsed between these ITC studies and ours. In a brief review that included eight studies examining attitudes of adult smokers to smoking restrictions in cars with children ${ }^{22}$, the authors found that support for such regulations generally increased with time, for example, from $63 \%, 45 \%$ and $51 \%$ of surveyed smokers in New South Wales (Australia) in 1994, 2000 and 2004, respectively, to $96 \%$ in New Zealand in 2007-2008 and 87\% in South Australia (Australia) in $2008^{22}$.

It is noteworthy to mention some differences observed in the prevalence of partial smoking rules and no-smoking rules in homes. For example, we observed that the higher the educational level, the higher the prevalence of partial bans in homes and the lower the prevalence of no rules. Also, the trend in the prevalence of the different rules differed by sex, age group, educational level and having children. The prevalence of no-smoking rules among participants who had children was almost half the prevalence of no-smoking rules among those who did not have children; conversely, the prevalence of partial bans was slightly higher among those who had children. In cars, such differences in prevalence are not remarkable, but we still observed some by educational level in a similar way to what we observed in homes. By CPD, the more the CPD smoked the more the prevalence of partial smoking rules and nosmoking rules; but the increase is two-fold for partial rules, whereas it is three-fold for no rules.

In our study, some of the factors that seem to affect smokers' support for smoke-free regulations in these private settings are: having higher educational level, having children, and having a non-smoker partner. Age seems to have a different effect on the support for smoking regulation in these settings; higher support for smoke-free homes was observed in younger people and higher support for smokefree cars in older people. Our data also showed that a total smoking ban in homes and cars was more likely among smokers with higher educational level, as shown by others ${ }^{23,24}$. This possibly indicates that the adoption of voluntary smoke-free rules among smokers follows diffusion of innovations theory (early adopters are those from more advantaged socioeconomic groups $)^{25}$. Although having children is taken into account in deciding to have both partial and total smoking bans in both settings, we observed that this factor was more important for total smoking bans. This finding has potential implications for the encouragement of smokers with partial smoking bans to move towards total smoking bans in their homes and cars. In addition, having a non-smoker partner was also associated with smoking bans in homes and cars, indicating the importance of social support in encouraging smoke-free environments in private settings. The social system is crucial for the diffusion of smoking bans, because decisions are made not only at the individual but also at the collective and societal level ${ }^{25}$, thus highlighting the importance of the interaction between the individual and the environment ${ }^{26}$.

The results show, however, that some improvements are needed to protect non-smokers from second-hand smoke exposure in private settings, especially vulnerable members of the population, such as children. It has been proven that partial smoking does not effectively protect non-smokers from second-hand tobacco smoke due to leaking smoke $\mathrm{e}^{27-29}$; furthermore, smoking in this context is still being promoted as a social norm. Furthermore, 
our findings demonstrate differences among smokers across the six EU MS in the attempt to implement smoking bans in private settings. Cross-country variation might reflect the level of denormalization of smoking in each country. Furthermore, differences may be related to the implementation status of smoke-free legislation, namely Article 8 of the FCTC, within each EU MS. While smoke-free legislation generally does not have wide jurisdiction over private settings, several studies have demonstrated the positive impact of smoke-free legislation on these unregulated places ${ }^{10,11,30-33}$. Comprehensive population-level policies may increase the prevalence of smoke-free environments in private settings ${ }^{33,34}$ by promoting awareness of the harmful effects of second-hand smoke exposure and the beneficial effects of reducing such exposure. Among the EU MS participating in our study, Germany and Poland are the countries were the least proportion of the population is protected by smoke-free laws regulating public places (national regulation is absent in Germany, while Poland has few public places where smoking is prohibited by national law $)^{35}$. When examining the prevalence of total or partial smoking bans by country, no clear pattern in relation to smoke-free environment legislation was observed; therefore, further longitudinal analyses are necessary to better explain the effects of smoke-free regulations and voluntary smoking bans in private settings. Also, studies should take into account the degree to which public regulations are enforced (overall and by country) and its relationship to other issues, such as social conditions that may affect smoking bans in private settings.

Disentangling the relationship and causal pathway between the awareness of second-hand smoke risks, the country-level climate of smoking denormalization, the degree of implementation of smoke-free legislation, and the individual opting for smoke-free homes and cars, are challenging issues that warrant further research. A comprehensive understanding of the factors associated with the voluntary implementation of smoke-free rules in private settings and the mechanism of impact on the population, can contribute to enhancing and tailoring interventions.

This study has some limitations. First, this is a survey conducted among smokers; thus, some information bias on smoking rules is possible due to social desirability and the general antismoking climate, with country variations across EU-MS. These results, therefore, cannot be generalised to all populations, such as non-smokers. Voluntary smokefree rules in homes and cars are, however, more frequent among non-smokers ${ }^{36-40}$. Second, survey questions on smoking rules in cars were asked specifically when children were present in the car, indicating that smoking may still occur in the absence of minors in the car. Third, the cross-sectional nature of the analysis precludes any inference about cause-effect relationships between the variables studied. Fourth, the use of self-reported information may introduce a source of bias that can affect the validity of our results. Nevertheless, self-reporting of smoking bans has been shown to have moderate to high correlation with measures of airborne nicotine, with excellent specificity and positive predictive values $^{29}$. Also, the use of questionnaires is extensive in population studies, because it allows reaching many people at low cost.

On the other hand, the study has many strengths, including a large sample size, the representativeness of the samples of smokers in each country, and the use of a common questionnaire, derived from the expansive ITC Project ${ }^{14}$. Moreover, the use of six EU MS with diverse geographical and sociodemographic characteristics provided variability in the implementation extent of smoke-free rules in private settings. These EU MS have different smoking prevalence and different stages of the tobacco epidemic (all of them at advanced stages, III or IV of the tobacco epidemic model) ${ }^{41}$. In addition, these countries share some legislation, such as the EU Tobacco Products Directive, whilst maintaining their own legislation. This study provides a baseline assessment for analysis of prospective data in successive waves of the EUREST-PLUS cohort study that will allow trends to be examined. The current analysis will further facilitate evaluation of changes in voluntary smoke-free rules in private settings, and other smoking-related variables, as a result of changes in legislation.

\section{CONCLUSIONS}

This study found that a quarter of smokers have a voluntary smoking ban at home and almost twothirds for cars in the presence of children. These figures, however, are far from satisfactory and 
highlight the need to promote public policies and interventions aimed at increasing the number of smoke-free homes and cars.

\section{*EUREST-PLUS consortium members:}

European Network on Smoking and Tobacco Prevention (ENSP), Belgium: Constantine I. Vardavas, Andrea Glahn, Christina N. Kyriakos, Dominick Nguyen, Katerina Nikitara, Cornel Radu-Loghin, Polina Starchenko University of Crete (UoC), Greece: Aristidis Tsatsakis, Charis Girvalaki, Chryssi Igoumenaki, Sophia Papadakis, Aikaterini Papathanasaki,

Manolis Tzatzarakis, Alexander I. Vardavas

Kantar Public (TNS), Belgium: Nicolas Bécuwe, Lavinia Deaconu, Sophie Goudet, Christopher Hanley, Oscar Rivière

Smoking or Health Hungarian Foundation (SHHF), Hungary: Tibor Demjén, Judit Kiss, Piroska A. Kovacs

Catalan Institut of Oncology (ICO); Bellvitge Biomedical Research Institute (IDIBELL), Spain: Esteve Fernández, Yolanda Castellano, Marcela Fu, Sarah 0. Nogueira, Olena Tigova

Kings College London (KCL), United Kingdom: Ann McNeill, Katherine East, Sara C. Hitchman

Cancer Prevention Unit and WHO Collaborating Centre for Tobacco Control, German Cancer Research Center (DKFZ), Germany: Ute Mons, Sarah Kahnert

National and Kapodistrian University of Athens (UoA), Greece: Yannis Tountas, Panagiotis Behrakis, Filippos T. Filippidis, Christina Gratziou, Paraskevi Katsaounou, Theodosia Peleki, Ioanna Petroulia, Chara Tzavara Aer Pur Romania, Romania: Antigona C. Trofor, Marius Eremia, Lucia Lotrean, Florin Mihaltan

European Respiratory Society (ERS), Switzerland; Goethe University Frankfurt, Germany: Gernot Rohde, Tamaki Asano, Claudia Cichon, Amy Far, Céline Genton, Melanie Jessner, Linnea Hedman, Christer Janson, Ann Lindberg, Beth Maguire, Sofia Ravara, Valérie Vaccaro, Brian Ward

Maastricht University, the Netherlands: Marc Willemsen, Hein de Vries, Karin Hummel, Gera E. Nagelhout

Health Promotion Foundation (HPF), Poland: Witold A. Zatoński, Aleksandra Herbeć, Kinga Janik-Koncewicz, Krzysztof Przewoźniak, Mateusz Zatoński University of Waterloo (UW); Ontario Institute for Cancer Research, Canada: Geoffrey T. Fong, Thomas K. Agar, Pete Driezen, Shannon Gravely, Anne C. K. Quah, Mary E. Thompson

\section{REFERENCES}

1. World Health Organization. Global Health Observatory data. http://www.who.int/gho/phe/secondhand_ smoke/en/. Accessed March, 2018.

2. World Health Organization. WHO Framework Convention on Tobacco Control. Geneva: World Health Organization; 2003.

3. WHO Framework Convention on Tobacco Control. Guidelines for implementation of Article 8. http:// www.who.int/fctc/guidelines/adopted/article_8/en/. Published, 2007. Accessed March, 2018.

4. Uang R, Hiilamo H, Glantz SA. Accelerated adoption of smoke-free laws after ratification of the World Health Organization Framework Convention on Tobacco Control. Am J Public Health. 2016;106:166-171. doi:10.2105/AJPH.2015.302872

5. International Agency for Research on Cancer. Evaluating the effectiveness of smoke-free policies. IARC Handbooks of Cancer Prevention, Tobacco Control. Vol. 13. Lyon, France: International Agency for Research on Cancer; 2009.
6. Gravely S, Giovino GA, Craig L, et al. Implementation of key demand-reduction measures of the WHO Framework Convention on Tobacco Control and change in smoking prevalence in 126 countries: an association study. Lancet Public Health. 2017;2:e166-e174. doi:10.1016/S2468-2667(17)30045-2

7. Eriksen MP. The Tobacco Atlas. 5th ed. Atlanta: American Cancer Society; 2015.

8. Raoof SA, Agaku IT, Vardavas CI. A systematic review of secondhand smoke exposure in a car: attributable changes in atmospheric and biological markers. Chron Respir Dis. 2015;12:120-131. doi:10.1177/1479972315575202

9. Orton S, Jones LL, Cooper S, Lewis S, Coleman T. Predictors of children's secondhand smoke exposure at home: a systematic review and narrative synthesis of the evidence. PLoS One. 2014;9:e112690. doi:10.1371/journal.pone.0112690

10. Monson E, Arsenault N. Effects of enactment of legislative (public) smoking bans on voluntary home smoking restrictions: a review. Nicotine Tob Res. 2017;19:141-148. doi:10.1093/ntr/ntw171

11. Fernández E, Fu M, Pérez-Ríos M, Schiaffino A, Sureda X, López MJ. Changes in Secondhand Smoke Exposure After Smoke-Free Legislation (Spain, 2006-2011). Nicotine Tob Res. 2017;19:1390-1394. doi:10.1093/ $\mathrm{ntr} / \mathrm{ntx} 040$

12. Fong GT, Cummings KM, Borland R, et al. The conceptual framework of the International Tobacco Control (ITC) Policy Evaluation Project. Tob Control. 2006;15 Suppl 3:iii3-iii11. doi:10.1136/tc.2005.015438

13. Official Journal of the European Union. Directive 2014/40/EU of the European Parliament and of the Council of 3 April 2014 on the approximation of the laws, regulations and administrative provisions of the Member States concerning the manufacture, presentation and sale of tobacco and related products and repealing Directive 2001/37/EC. 2014;OJ L 127, 57:1-38. http:// data.europa.eu/eli/dir/2014/40/oj. Accessed March, 2018.

14. Vardavas CI, Bécuwe N, Demjén T, et al. Study Protocol of European Regulatory Science on Tobacco (EURESTPLUS): Policy implementation to reduce lung disease. Tobacco Induced Diseases. 2018;(Suppl 2:A2). doi:10.18332/tid/93305

15. Fong GT, Thompson ME, Boudreau C, et al. The Conceptual Model and Methods of Wave 1 (2016) of the EUREST-PLUS ITC 6 European Countries Survey. Tobacco Induced Diseases. 2018;16(Suppl 2:A3). doi:10.18332/tid/99881

16. Heatherton TF, Kozlowski LT, Frecker RC, Rickert W, Robinson J. Measuring the heaviness of smoking: using self-reported time to the first cigarette of the day and number of cigarettes smoked per day. Br J Addict. 1989;84:791-799. doi:10.1111/j.1360-0443.1989.tb03059.x 
17. Borland R, Yong HH, Cummings KM, Hyland A, Anderson S, Fong GT. Determinants and consequences of smoke-free homes: findings from the International Tobacco Control (ITC) Four Country Survey. Tob Control. 2006;15 Suppl 3:iii42-iii50. doi:10.1136/tc.2005.012492

18. Hyland A, Hassan LM, Higbee C, et al. The impact of smokefree legislation in Scotland: results from the Scottish ITC: Scotland/UK longitudinal surveys. Eur J Public Health. 2009;19:198-205. doi:10.1093/eurpub/ckn141

19. King BA, Hyland AJ, Borland R, McNeill A, Cummings KM. Socioeconomic variation in the prevalence, introduction, retention, and removal of smoke-free policies among smokers: findings from the International Tobacco Control (ITC) Four Country Survey. Int J Environ Res Public Health. 2011;8:411-434. doi:10.3390/ijerph8020411

20. Mons U,Nagelhout GE,Allwright S, etal.Impact of national smoke-free legislation on home smoking bans: findings from the International Tobacco Control Policy Evaluation Project Europe Surveys. Tob Control. 2013;22:e2-e9. doi:10.1136/tobaccocontrol-2011-050131

21. Hitchman SC, Guignard R, Nagelhout GE, et al. Predictors of car smoking rules among smokers in France, Germany and the Netherlands. Eur J Public Health. 2012;22 Suppl 1:17-22. doi:10.1093/eurpub/ ckr200

22. Thomson G, Wilson N. Public attitudes to laws for smoke-free private vehicles: a brief review. Tob Control. 2009;18:256-261. doi:10.1136/tc.2008.027672

23. Mills AL, Messer K, Gilpin EA, Pierce JP. The effect of smoke-free homes on adult smoking behavior: a review. Nicotine Tob Res. 2009;11:1131-1141. doi:10.1093/ntr/ntp122

24. Montreuil A, Hanusaik N, Cantinotti M, et al. Social disparities in children's exposure to secondhand smoke in privately owned vehicles. Tob Control. 2017;26:663668. doi:10.1136/tobaccocontrol-2016-053347

25. Rogers EM. Diffusion of Innovations. 5th ed. New York, NY: Simon \& Schuster; 2003.

26. Hull CL. Principles of behavior - An introduction to behavior theory. New York: Appleton-Century-Crofts, Inc.; 1943.

27. Nebot M, Lopez MJ, Ariza C, et al. Impact of the Spanish smoking law on exposure to secondhand smoke in offices and hospitality venues: before-and-after study. Environ Health Perspect. 2009;117:344-347. doi:10.1289/ehp.11845

28. Fernández E, Fu M, Pascual JA, et al. Impact of the Spanish smoking law on exposure to secondhand smoke and respiratory health in hospitality workers: a cohort study. PLoS One. 2009;4:e4244. doi:10.1371/journal.pone.0004244

29. Arechavala T, Continente X, Perez-Rios M, et al.
Second-hand smoke exposure in homes with children: assessment of airborne nicotine in the living room and children's bedroom. Tob Control. 2018;27:399-406. doi:10.1136/tobaccocontrol-2017-053751

30. Haw SJ, Gruer L. Changes in exposure of adult nonsmokers to secondhand smoke after implementation of smoke-free legislation in Scotland: national cross sectional survey. BMJ. 2007;335:549. doi:10.1136/bmj.39315.670208.47

31. Cheng KW, Okechukwu CA, McMillen R, Glantz SA. Association between clean indoor air laws and voluntary smokefree rules in homes and cars. Tob Control. 2015;24:168-174. doi:10.1136/tobaccocontrol-2013-051121

32. Sureda X, Martínez-Sánchez JM, Fu M, et al. Impact of the Spanish smoke-free legislation on adult, non-smoker exposure to secondhand smoke: cross-sectional surveys before (2004) and after (2012) legislation. PLoS One. 2014;9:e89430. doi:10.1371/journal.pone.0089430

33. Ferketich AK, Lugo A, La Vecchia C, et al. Relation between national-level tobacco control policies and individual-level voluntary home smoking bans in Europe. Tob Control. 2016;25:60-65. doi:10.1136/tobaccocontrol-2014-051819

34. Thomson G, Wilson N, Howden-Chapman P. Population level policy options for increasing the prevalence of smokefree homes. J Epidemiol Community Health. 2006;60:298-304. doi:10.1136/jech.2005.038091

35. World Health Organization. WHO report on the global tobacco epidemic, 2017: monitoring tobacco use and prevention policies. Geneva: World Health Organization; 2017.

36. Hood NE, Wewers ME, Ferketich AK, Klein EG, Pirie P. Predictors of voluntary home-smoking restrictions and associations with an objective measure of in-home smoking among subsidized housing tenants. Am J Health Promot. 2013;28:97-104. doi:10.4278/ajhp.120816-QUAN-399

37. Ayo-Yusuf OA, Olufajo O, Agaku IT. Exposure to secondhand smoke and voluntary adoption of smoke-free home and car rules among non-smoking South African adults. BMC Public Health. 2014;14. doi:10.1186/1471-2458-14-580

38. King BA, Patel R, Babb SD. Prevalence of smokefree home rules--United States, 1992-1993 and 2010-2011. MMWR Morb Mortal Wkly Rep. 2014;63:765-769.

39. Milcarz K, Bak-Romaniszyn L, Kaleta D. Environmental Tobacco Smoke Exposure and Smoke-Free Rules in Homes among Socially-Disadvantaged Populations in Poland. Int J Environ Res Public Health. 2017;14. doi:10.3390/ijerph14040447

40. Diez-Izquierdo A, Lidon-Moyano C, Martin-Sanchez JC, et al. Smoke-free homes and attitudes towards banning smoking in vehicles carrying children in Spain (2016). Environ Res. 2017;158:590-597. 
doi:10.1016/j.envres.2017.07.012

41. Thun M, Peto R, Boreham J, Lopez AD. Stages of the cigarette epidemic on entering its second century. Tob Control. 2012;21:96-101. doi:10.1136/tobaccocontrol-2011-050294

ACKNOWLEDGEMENTS

We want to thank the ITC Project Team at the University of Waterloo, particularly Pete Driezen, for their support in ITC dataset management and preliminary data analysis.

CONFLICTS OF INTEREST

The authors declare that they have no competing interests, financial or otherwise, related to the current work. K. Przewoźniak reports grants and personal fees from the Polish League Against Cancer, outside the submitted work. C. I. Vardavas reports that he is the Strategic Development Editor of TID and that there are no conflicts of interest with this current work. The rest of the authors have also completed and submitted an ICMJE form for disclosure of potential conflicts of interest.

\section{FUNDING}

The EUREST-PLUS project has received funding from the European Union's Horizon 2020 research and innovation programme under grant agreement No 681109 (CIV) and the University of Waterloo (GTF). Additional support was provided to the University of Waterloo by the Canadian Institutes of Health Research (FDN-148477). GTF was supported by a Senior Investigator Grant from the Ontario Institute for Cancer Research. Authors EF, MF, OT and YC are supported by the Ministry of Universities and Research, Government of Catalonia (2017SGR319). EF was supported by the Instituto de Salud Carlos III, Government of Spain (INT16/00211 and INT17/00103), co-funded by the European Regional Development Fund (FEDER).

PROVENANCE AND PEER REVIEW

Commissioned; externally peer reviewed. 